\title{
TEORES FOLIARES DE CÁLCIO E BORO NO FEIJOEIRO INFLUENCIADOS PELO TEMPO DE MOLHAMENTO DA ÁGUA DE IRRIGAÇÃO POR PIVÔ-CENTRAL
}

\section{Pedro Marques da Silveira ${ }^{1 *}$, Luís Fernando Stone ${ }^{1}$, João Pedro Miranda Caetano ${ }^{2}$, Mateus Miranda Caetano ${ }^{2}$, Pedro Henrique Lopes Sarmento ${ }^{1}$, Luciene Frões Camarano de Oliveira ${ }^{1}$.}

\author{
${ }^{1}$ Embrapa Arroz e Feijão, Santo Antônio de Goiás, 75375-000, Goiás, Brasil \\ ${ }^{2}$ Faculdade do Noroeste de Minas, Paracatú, 38600-000, Minas Gerais, Brasil
}

Autor para correspondência: Pedro Marques da Silveira; e-mail: pedro.silveira@embrapa.br

\begin{abstract}
Resumo
A lavagem de nutrientes das folhas de plantas pela água das chuvas é um fato reconhecido no meio agronômico. Com o advento da prática de irrigação de grandes áreas, em diferentes culturas, o fenômeno ainda não mereceu a atenção necessária. O objetivo do estudo foi determinar o conteúdo foliar de cálcio e boro no limbo foliar do feijão, em razão do tempo de molhamento da planta, ocasionado pelo tempo de operação do pivô-central, em quatro diferentes pontos de localização no campo, ao longo do raio do equipamento. $\mathrm{O}$ estudo foi conduzido no município de Cristalina - GO, em uma lavoura de feijão, de propriedade particular, irrigada pelo sistema de irrigação por pivô-central. A cultivar utilizada foi a BRS FC402. No início do florescimento da cultura, foram coletadas amostras de folhas da planta para análise foliar. Até aquele momento de coleta das amostras, a cultura tinha recebido $150 \mathrm{~mm}$ de água de irrigação. As amostras foram coletadas em quatro pontos equidistantes ao longo do raio do pivô-central, com quatro repetições. Foram analisados os teores de cálcio e boro no limbo foliar. Os teores desses nutrientes foram tanto maiores quanto menores os tempos de molhamento foliar pela água de irrigação.
\end{abstract}

Palavras-chave: Lavagem de nutrientes; Nutrição mineral; Phaseolus vulgaris

\section{LEAF CONTENTS OF CALCIUM AND BORON IN THE BEANSTALK INFLUENCED BY THE WETTING TIME OF THE IRRIGATION WATER BY CENTRAL PIVOT}

\begin{abstract}
The washing of nutrients from plant leaves by rainwater is a recognized fact in the agronomic environment. With the advent of the practice of irrigating large areas, in different cultures, the phenomenon has not yet received the necessary attention. The objective of the study was to determine the leaf content of calcium and boron in the leaf blade of the common bean, due to the time of wetting of the plant, caused by the time of operation of the central pivot, in four different points of location in the field, along the equipment radius. The study was conducted in the municipality of Cristalina - GO, in a common bean field, privately owned, irrigated by the central pivot irrigation system. The cultivar used was BRS FC402. At the beginning of the flowering of the culture, samples of plant leaves were collected for leaf analysis. Until that moment of sample collection, the culture had received $150 \mathrm{~mm}$ of irrigation water. The samples were collected at four equidistant points along the central pivot radius, with four replications. The calcium and boron contents in the leaf blade were analyzed. The contents of these nutrients were greater the shorter the leaf wetting times by irrigation water.
\end{abstract}

Keywords: Nutrient washing; Mineral nutrition; Phaseolus vulgaris. 


\section{Introdução}

A terceira época de plantio de feijão, feijão de outono-inverno, foi lançada em 1980, em São Paulo, dois anos após o lançamento do equipamento pivôcentral no Brasil. Hoje, caracteriza-se como uma safra muito importante, reguladora de estoque, correspondendo nos últimos três anos a 21,9\% da produção brasileira (IBGE, 2019) com produtividades acima de $3.000 \mathrm{~kg} \mathrm{ha}^{-1} \mathrm{e}$, totalmente, dependente da irrigação.

O sistema de irrigação pivô-central consiste, basicamente, de aspersores difusores, montados sobre uma linha lateral, suportada por uma série de torres, que se movimentam sobre rodas ao redor do ponto central da área irrigada, denominado Ponto do pivô. Dado a esse tipo de funcionamento, o tempo de molhamento e a intensidade de aplicação de água variam ao longo do raio do equipamento.

Um fenômeno conhecido de há muito é a saída de nutrientes das folhas e outras partes aéreas das plantas, por efeito da lavagem por água de chuva, orvalho ou neblina (CAMARGO, 1970).

Já em 1959, Wittwer e Teubner (1959) relataram que a quantidade de nutrientes e compostos orgânicos que sai das folhas por lavagem era realmente grande e, em condições naturais, ela poderia ser um importante fator regulador da composição mineral e orgânica das partes aéreas das plantas.

Segundo Camargo (1970), as perdas por lavagem, em 24 horas, podem chegar a $80-90 \%$ do conteúdo de potássio e $50-60 \%$ do conteúdo de cálcio das folhas maduras e que a lavagem varia com a natureza do material lavado e com a espécie de planta. E, na mesma planta, ela varia com a idade e o tipo das folhas. Ainda conforme o autor, o mecanismo de lavagem parece tratar-se de um processo físico, de difusão e trocas, e que a fonte maior de nutrientes laváveis são os nutrientes que ainda estão se translocando apoplásticamente.

Mecklenburg e Tukey Jr. (1964) relataram que 30\% a $40 \%$ do cálcio recentemente absorvido pela raiz foi lixiviado pelas folhas do feijoeiro durante um período de lixiviação de quatro dias.

Existem três hipóteses para a lixiviação de cálcio das folhas (MECKLENBURG; TUKEY JR.; MORGAN, 1966). A primeira é que a lixiviação ocorre pela troca do cálcio presente na cutícula e locais de troca da parede celular por hidrogênio da solução de lixiviação. A água na superfície da folha dissolve o $\mathrm{CO}_{2}$ do ar para formar o ácido carbônico. O ácido carbônico se dissocia e o hidrogênio liberado intercambia com cátions nos locais de troca da cutícula para formar carbonatos alcalinos, que permanecem na solução lixiviada ou são precipitados na superfície da folha. A segunda hipótese é que a lixiviação ocorre por difusão de íons da corrente de translocação dentro da folhagem para a solução de lixiviação e a terceira é a combinação de troca e difusão. Os ectodesmas, que aparecem como numerosos fios protoplasmáticos entre as células epidérmicas e o exterior da folha, também podem desempenhar um papel importante neste processo.

As folhas maduras são mais suscetíveis à lixiviação de metabólitos orgânicos e inorgânicos do que as folhas jovens. O cálcio é facilmente lixiviado do reservatório de cálcio trocável nos tecidos maduros. No entanto, tecidos jovens em crescimento vigoroso acumulam cálcio dentro das células e paredes celulares, de onde não é facilmente lixiviado, reduzindo assim a quantidade de cálcio trocável disponível para lixiviação (MECKLENBURG; TUKEY JR.; MORGAN, 1966).

$O$ cálcio $(\mathrm{Ca})$ e o boro (B) são elementos essenciais para o feijoeiro. Segundo Fageria et al. (2015), dentre os micronutrientes, o B merece atenção especial na nutrição do feijão-comum, devido ao seu papel fundamental em processos fisiológicos na planta, os quais impactam diretamente na produtividade, tais como: germinação dos grãos de pólen e o crescimento do tubo polínico; a formação da parede celular; e translocação de carboidratos, que são essenciais para a produção de grãos. Além disso, o B pode afetar o nível de $\mathrm{Ca}^{2+}$ no citosol e o processo de estabilização de proteínas (GONZÁLEZ-FONTES et al., 2014).

O objetivo do estudo foi determinar o conteúdo foliar de cálcio e boro no limbo foliar do feijão em razão do tempo de molhamento da planta (lavagem), ocasionado pelo tempo de operação do pivô-central em quatro diferentes pontos de localização no campo, ao longo do raio do equipamento.

\section{Material e Métodos}

O estudo foi conduzido no município de Cristalina GO, em uma lavoura de feijão, de propriedade particular, irrigada pelo sistema de irrigação por pivôcentral. A cultivar utilizada foi a BRS FC402, de grão carioca, semeada em 29/04/2020. A adubação foi de 200 $\mathrm{kg} \mathrm{ha}^{-1}$ de MAP no plantio e $150 \mathrm{~kg} \mathrm{ha}^{-1} \mathrm{de} \mathrm{KCl}+150$ $\mathrm{kg} \mathrm{ha}^{-1}$ de ureia na fase vegetativa.

$\mathrm{O}$ pivô-central utilizado apresentava a seguinte ficha técnica: área irrigada $=103,54 \mathrm{ha}$, comprimento do pivô $=565,73 \mathrm{~m}$, distância da base a última torre $=$ $538,64 \mathrm{~m}$, tempo para uma volta completa a $100 \%=$ 12,63 horas, velocidade da última torre a $100 \%=268$ $\mathrm{m} / \mathrm{h}$, lâmina bruta por volta a $100 \%=4,81 \mathrm{~mm}$, diâmetro de alcance do difusor $=6,0 \mathrm{~m}$

No início do florescimento da cultura, foram coletadas amostras de folhas de feijão para análise foliar. Coletou-se o terceiro trifólio completamente desenvolvido a partir do ápice da planta, de 30 plantas 
diferentes por área homogênea, conforme recomendação de Carvalho et al. (2019). Até o momento de coleta das amostras, a cultura tinha recebido $150 \mathrm{~mm}$ de água de irrigação. As amostras foram coletadas em quatro pontos equidistantes ao longo do raio do pivô-central, com quatro repetições. Foram analisados os teores dos nutrientes $\mathrm{Ca}$ e B, segundo a metodologia da Embrapa (2009).

Os pontos de coleta foram: (P1) $75 \mathrm{~m}$, (P2) $200 \mathrm{~m}$, (P3) $325 \mathrm{~m}$ e (P4) $450 \mathrm{~m}$ da base do pivô-central, respectivamente, $13 \%, 35 \%, 57 \%$ e $80 \%$ do raio do equipamento. A fórmula para calcular a velocidade em cada ponto do pivô, em um determinado ajuste do percentímetro, é:

$$
\mathrm{v}=2 \pi \mathrm{r} / \mathrm{T}
$$

em que:

$\mathrm{v}=$ velocidade no ponto, $\mathrm{em} \mathrm{m} \mathrm{h}^{-1}$

$\mathrm{r}=$ distância do ponto considerado ao ponto pivô, em m

$\mathrm{T}$ = tempo para uma volta completa no ajuste do percentímetro considerado, em $\mathrm{h}$.

Assim, o tempo de molhamento em cada ponto do pivô é calculado por:

em que:

$$
\mathrm{t}=\mathrm{d} / \mathrm{v}
$$

$\mathrm{t}=$ tempo de molhamento em cada ponto do pivô, em $\mathrm{h}$

$\mathrm{d}=$ diâmetro molhado do difusor, em $\mathrm{m}$

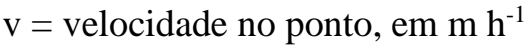

Juntando as duas equações, tem-se:

$$
\mathrm{t}=\mathrm{dT} / 2 \pi \mathrm{r}
$$

Tempo de molhamento para aplicação de 4,81 mm de água:

$(450 \mathrm{~m}) \mathrm{T}=6,0 \times 12,63 / 2 \times 3,1416 \times 450=0,026801 \mathrm{~h}$ $(325 \mathrm{~m}) \mathrm{T}=6,0 \times 12,63 / 2 \times 3,1416 \times 325=0,037109 \mathrm{~h}$ $(200 \mathrm{~m}) \mathrm{T}=6,0 \times 12,63 / 2 \times 3,1416 \times 200=0,060303 \mathrm{~h}$ $(75 \mathrm{~m}) \mathrm{T}=6,0 \times 12,63 / 2 \times 3,1416 \times 75=0,160809 \mathrm{~h}$

A lâmina total aplicada foi de $150 \mathrm{~mm}$ até início do florescimento. Desse modo, o tempo total de molhamento foi:

$(450 \mathrm{~m}) \mathrm{T}=0,026801(150 / 4,81)=0,84 \mathrm{~h}$

$(325 \mathrm{~m}) \mathrm{T}=0,037109(150 / 4,81)=1,16 \mathrm{~h}$

$(200 \mathrm{~m}) \mathrm{T}=0,060303(150 / 4,81)=1,88 \mathrm{~h}$

$(75 \mathrm{~m}) \mathrm{T}=0,160809(150 / 4,81)=5,01 \mathrm{~h}$

Os dados foram submetidos à análise de variância e à análise de regressão.

\section{Resultados e Discussão}

A Tabela 1 apresenta os teores de cálcio e boro no limbo foliar do feijoeiro em razão do tempo de molhamento pela água de irrigação por pivô-central. Houve efeito significativo dos tratamentos de molhamento foliar sobre os teores de Ca e B no limbo foliar do feijoeiro.

Tabela 1. Teores de cálcio (Ca) e boro (B) no limbo foliar do feijoeiro, BRS FC402, no início da floração, em razão do tempo de molhamento pela água de irrigação por pivô-central.

\begin{tabular}{ccc}
$\begin{array}{c}\text { Molhamento foliar } \\
\text { (hora) }\end{array}$ & $\begin{array}{c}\text { Teor de Ca } \\
\left(\mathbf{g ~ k g ~}^{-\mathbf{1}}\right)\end{array}$ & $\begin{array}{c}\text { Teor de B } \\
(\mathbf{m g ~ k g}\end{array}$ \\
\hline 5,01 & 14,2 & 29,1 \\
1,88 & 14,2 & 31,0 \\
1,16 & 16,0 & 33,9 \\
0,84 & 18,1 & 36,7 \\
\hline C.V. $(\%)$ & 14,0 & 9,90 \\
\hline DMS & 3,43 & 3,17 \\
\hline \multicolumn{3}{|c}{ ANOVA } \\
QM trat & 13,5 & 43,6 \\
QM res & 2,66 & 2,28 \\
Fc & 5,08 & 19,0 \\
$P$ & 0,01684 & 0,00007 \\
\hline
\end{tabular}

Realizou-se estudo de regressão entre as variáveis, de acordo com a Figura 1 para cálcio e Figura 2 para boro. Os teores de Ca diminuíram de forma quadrática com o aumento do tempo de molhamento foliar ( $\mathrm{p}<$ $0,01684)$. Ocorreu o mesmo para Boro ( $\mathrm{p}<0,00007)$. Logo, o aumento do tempo de molhamento foliar causou lavagem desses dois nutrientes na folha do

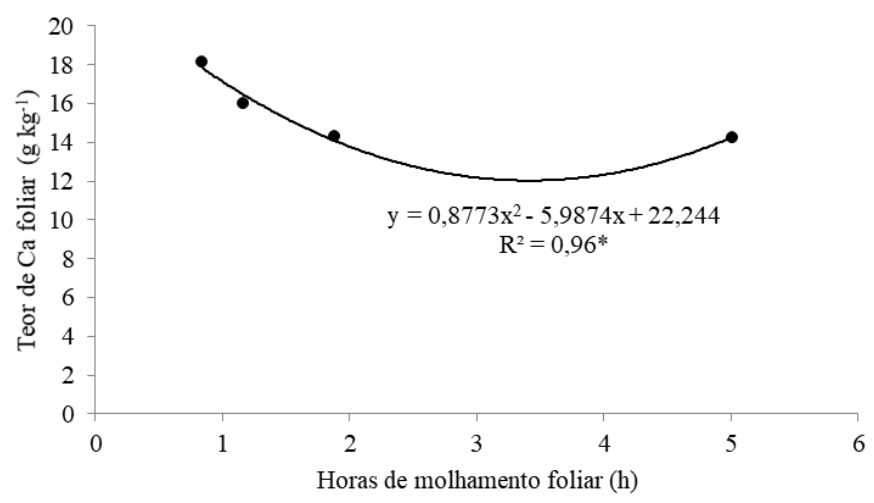

feijoeiro.

Figura 1. Teores de cálcio $(\mathrm{Ca})$ no limbo foliar do feijoeiro, no início da floração, em razão do tempo de molhamento pela água de irrigação por pivô-central. 


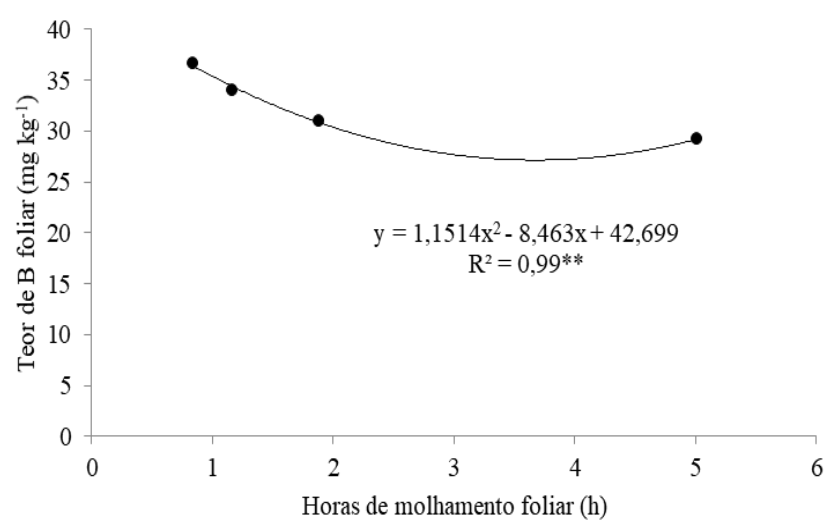

Figura 2. Teores de boro (B) no limbo foliar do feijoeiro, no início da floração, em razão do tempo de molhamento pela água de irrigação por pivô-central.

Conforme Camargo (1970), o Ca é considerado um nutriente moderadamente lavável na folha e, quanto mais prolongado o período de lavagem, maior é a quantidade de metabolitos extraídos da folhagem, o que corrobora os resultados encontrados.

Nable e Moody (1992) constataram que chuva moderada diminuiu substancialmente a concentração e o conteúdo de $\mathrm{B}$ em folhas jovens de trigo.

De acordo com Mecklenburg et al. (1966), o orvalho e a chuva leve de longa duração são mais eficazes na lixiviação de metabólitos do que a chuva forte de curta duração. Esses autores constataram que o volume da solução de lixiviação teve pouca influência sobre a lixiviação do Ca das folhas do feijoeiro, o que pode ser explicado pela hipótese de troca e difusão. Com base nessa hipótese, o volume da solução de lixiviação só precisa ser suficiente para molhar a superfície foliar da folha, e o volume adicional aumentaria apenas ligeiramente a troca, talvez não o suficiente para ser detectado. Essa pode ser a razão da resposta dos teores foliares de $\mathrm{Ca}$ e $\mathrm{B}$ ao tempo de molhamento se ajustar a modelos quadráticos.

Os valores encontrados para Ca e B estão dentro das faixas de valores observados por Mesquita (2013), para a região, em um estudo de avaliação nutricional e normas DRIS para o feijoeiro irrigado em Cristalina, Goiás, envolvendo mais de 50 amostras de plantas.

$\mathrm{Na}$ Figura 3, são apresentados os teores de $\mathrm{Ca}$ ao longo do raio do pivô-central, considerando os pontos de coleta das amostras de plantas, os quais condicionaram os diferentes tempos de molhamento foliar. De modo similar, os teores de Ca na folha foram maiores quanto maior a distância da planta em relação a base (ponto) do pivô. Comportamento semelhante acorreu também para o B (Figura 4).

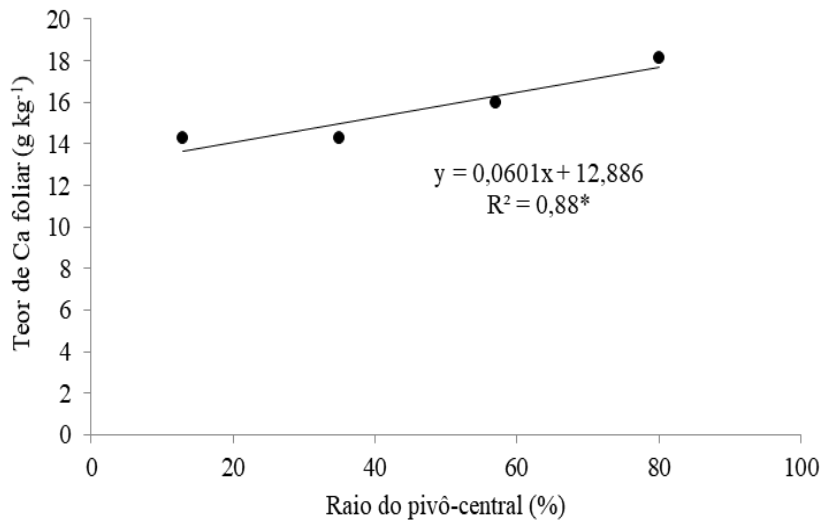

Figura 3. Teores de cálcio (Ca) no limbo foliar do feijoeiro, no início da floração, em razão da localização da planta, em percentagem $(\%)$ do raio do pivô-central.

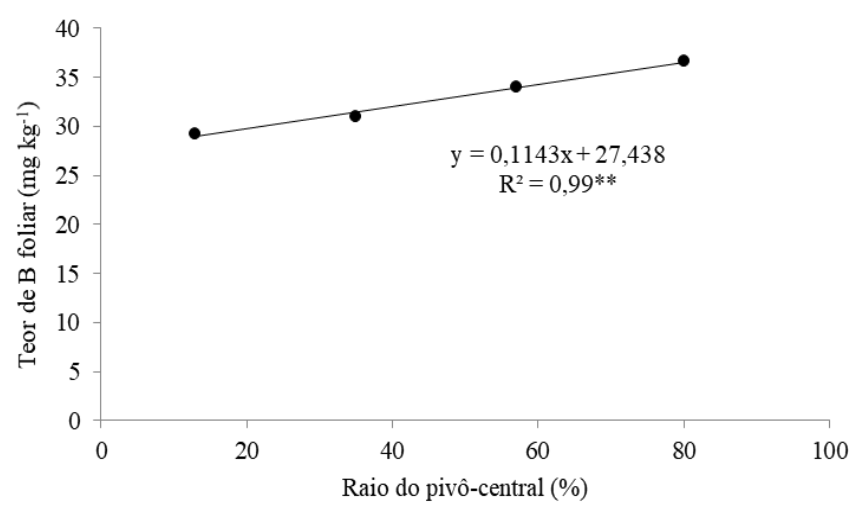

Figura 4. Teores de boro (Bo) no limbo foliar do feijoeiro, no início da floração, em razão da localização da planta, em percentagem (\%) do raio do pivô-central.

Camargo (1970) relatou que chuvas finas e prolongadas lavam muito maior quantidade de nutrientes que as pesadas e rápidas, de mesma precipitação. É o que acontece com a aplicação de água ao longo do raio do pivô-central, em que a intensidade de aplicação de água tende a aumentar na medida em que se afasta do centro do pivô-central (ALVES JUNIOR et al., 2010), para compensar o menor tempo de irrigação, para mesma precipitação.

Pela análise dos resultados, pode-se inferir que a lavagem de nutrientes, no ciclo total da cultura, deve ser maior, visto que, as coletas de plantas para as análises foliares foram realizadas no início do florescimento (VELOSO et al., 2003) quando haviam sido aplicados somente $150 \mathrm{~mm}$ de água de irrigação. Logo, mais irrigações foram feitas para completar o ciclo da cultura.

Nesse prisma, Mantovani et al. (2012) estimaram um requerimento total de água de irrigação para maior produtividade de feijão em Cristalina - GO de $418 \mathrm{~mm}$. Uma outra inferência é que, de modo contrário a esse pivô-central de 100 hectares, as perdas por lavagem podem ser menores, em caso de pivô-centrais menores, 
por ser menor a diferença no tempo de molhamento da planta pela água de irrigação, ao longo do raio do pivôcentral. A área média de pivôs-centrais em Cristalina GO é de 77 hectares (ANA, 2019).

A lixiviação foliar compete com sucesso com o metabolismo celular por cátions trocáveis e outros metabólitos que estão sendo exportados, podendo-se causar deficiência nutricional nas plantas durante períodos prolongados de chuva (MECKLENBURG et al., 1966).

Nesse diapasão, Mecklenburg e Tukey Jr. (1964) relataram que a lixiviação foliar de $\mathrm{Ca}$ reduziu o acúmulo de matéria seca do caule e folhagem do feijoeiro. A falta de cálcio e boro pode interferir na germinação do grão de pólen e no crescimento do tubo polínico e, consequentemente, causar a redução do número de sementes por vagem (MARSCHNER, 1995).

\section{Conclusões}

Os teores de cálcio e boro no limbo foliar do feijoeiro foram tanto maiores quanto menores os tempos de molhamento pela água de irrigação.

Os teores de cálcio e boro no limbo foliar do feijoeiro aumentaram na medida em que as plantas estavam mais distantes da base (ponto) do pivô-central.

\section{Referências}

ALVES JUNIOR, J.; SILVEIRA, P. M.; STONE, L. F.; CUNHA, P. C. R. Retenção de água pelo dossel do feijoeiro irrigado por aspersão. Irriga, Botucatu. v.15, n.1, p. 90-97, 2010.

ANA. Levantamento da agricultura irrigada por pivôs centrais no Brasil/Agência Nacional de Águas, Embrapa Milho e Sorgo. 2. ed. - Brasília: ANA, 2019. $47 \mathrm{p}$.

CAMARGO, P. N. Princípios de nutrição foliar. Editora Agronômica Ceres, 1970. 118p.

CARVALHO, M, C, S.; NUNES, R. S.; NASCENTE, A. S.; SILVEIRA, P. M. Nutrição e adubação do feijãocomum na região do Cerrado. In: FLORES, R. A.; CUNHA, P. P.; MARCHÃO, R. L.; MORAES, M. F. (Eds.). Nutrição e adubação de grandes culturas na região do Cerrado. Goiânia, GO: UFG, 2019. p.325367.

EMBRAPA. Manual de Análises Químicas de Solos, Plantas e Fertilizantes, 2. ed. 2009, 624 p.

FAGERIA, N. K.; STONE, L. F.; BAETA, A. B.; CARVALHO, M. C. S. Nutrição mineral do feijoeiro,
1. ed. Brasília, DF: Embrapa Informação Tecnológica, 2015.394p.

GONZÁLEZ-FONTES, A.; NAVARRO-GOCHICOA, M. T.; CAMACHO-CRISTÓBAL, J. J.; HERRERARODRÍGUEZ, M. B.; QUILES-PANDO, C.; REXACH, J. Is $\mathrm{Ca}^{2+}$ involved in the signal transduction pathway of boron deficiency? New hypotheses for sensing boron deprivation. Plant Science, v. 217-218, p. 135-139, 2014.

IBGE. Levantamento Sistemático da Produção Agrícola, 2019.

MANTOVANI, E. C.; MONTES, P. R. P.; VIEITRA, G. H. S.; RAMOS, M. M.; SOARES, A. A. Estimativa de produtividade da cultura do feijão irrigado em Cristalina-GO, para diferentes lâminas de irrigação como função da uniformidade de aplicação. Engenharia Agrícola, Jaboticabal. v. 32, n. 1, p.110120, 2012.

MARSCHNER, H. Mineral nutrition of higher plant. 2. ed. New York: Academic Press, 1995. 889 p.

MECKLENBURG, R. A.; TUKEY JR., H. B.; MORGAN, J. V. A mechanism for the leaching of calcium from foliage. Plant Physiology, v. 41, n. 4, p. 610-613, 1966.

MECKLENBURG, R. A.; TUKEY JR.; H. B. Influence of foliar leaching on root uptake and translocation of calcium-45 to the stems and foliage of Phaseolus vulgaris. Plant Physiology, v. 39, n. 4, p. 533-536, 1964.

MESQUITA, M. A. M. Avaliação Nutricional e normas DRIS para o feijoeiro irrigado em Cristalina, Goiás. Universidade Federal de Goiás, 2013, 109p. (Tese de Doutorado).

NABLE, R. O.; MOODY, D. B. Effects of rainfall on the use of foliar analysis for diagnosing boron toxicity in field-grown wheat. Plant and Soil, v. 140, n. 2, p. 311-314, 1992.

VELOSO, C. A. C.; ARAUJO, S. M. B.; VIEGHAS, I. J. M.; OLIVEIRA, R. C. Amostragem de plantas para análise química. Embrapa Amazônia Oriental. 2003, $4 \mathrm{p}$.

WITTWER, S. H.; TEUBNER, F. G. Foliar absorption of mineral nutrients. Anual Review of Plant Physiology. p.10-143, 1959. 